\title{
Light-front time picture of few-body systems
}

\author{
T. Frederico ${ }^{a}$, J. H. O. Sales ${ }^{b}$, B. V. Carlson ${ }^{a}$ and, P. U. Sauer . $^{c}$ \\ ${ }^{a}$ Dep. Física Instituto Tecnológico de Aeronáutica, \\ Centro Técnico Aeroespacial, 12.228-900 \\ São José dos Campos, São Paulo, Brazil. \\ ${ }^{b}$ Instituto de Física Teórica-UNESP, 01405-900 \\ São Paulo, Brazil. \\ ${ }^{c}$ Institute for Theoretical Physics, University Hannover \\ D-30167 Hannover, Germany
}

\begin{abstract}
The projection of the four-dimensional two-body Bethe-Salpeter equation at the light-front hypersurface is discussed. Elimination of the relative time is performed using a quasi-potential expansion, which is shown to be equivalent to an infinite set of coupled equations for the Green's functions of the different light-front Fock-states.
\end{abstract}

\section{INTRODUCTION}

The field theoretical Bethe-Salpeter equation (BSE) in the ladder approximation can be used as starting point to model bound-states of relativistic few-body systems. The light-front time dynamics [1] leads to the representation of the two-body BSE in which an effective mass squared operator arises and has as an eigenfunction the lowest Fockstate component (valence) of the wave-function [2,3]. The BSE is equivalent to an infinite set of coupled equations for Green's functions with different number of particles, which also allows a consistent truncation of the light-front Fock-space. This set of coupled equations comes from a quasi-potential expansion of the BSE on the light-front [2-4]. The relative light-front time between the particles is eliminated in favor of a global time.

The two-body bound-state light-front equation with the lowest order kernel [5] (truncation of the virtual intermediate states up to three-particles) was applied to study bosonic $[6]$ and fermionic $[7,8]$ systems. Nowadays, there is a renewed interest in studying the structure of few-body bound-states at the light-front time $x^{+}=t+z=0$, due to the possibility of probing hadrons and few-nucleon systems at short distances [9].

We perform the quasi-potential reduction of two-body BSE's and present the coupled set of equations for the light-front Green's functions for bosonic and fermionic models, with the interaction Lagrangian respectively given by, $\mathcal{L}_{I}^{B}=g_{S} \phi_{1}^{\dagger} \phi_{1} \sigma+g_{S} \phi_{2}^{\dagger} \phi_{2} \sigma$ and $\mathcal{L}_{I}^{F}=g_{S} \bar{\Psi} \Psi \sigma$, where $\phi_{1}, \phi_{2}$ and $\sigma$ are the bosonic fields and $\Psi$ is the fermion field in the Yukawa model.

\section{GLOBAL $X^{+}$-TIME AND QUASI-POTENTIAL REDUCTION}

The starting point is the BSE in relativistic field theory for the two-body transition matrix, $T(K)$, with total four-momentum $K$ and two-body irreducible interaction $V(K)$ :

$$
T(K)=V(K)+V(K) G_{0}(K) T(K) .
$$

Here, we neglect self-energy terms in the disconnected Green's function.

We begin by discussing the elimination of the relative $x^{+}$-time in (1) for a two-boson system [2]. The free Green's function expressed in terms of light-front kinematical momenta, e.g., $k_{i}=\left(k_{i}^{-}:=k_{i}^{0}-k_{i}^{3}, k_{i}^{+}:=k_{i}^{0}+k_{i}^{3}, \vec{k}_{i \perp}\right)$, is:

$$
\left\langle k_{1}^{\prime-}\left|G_{0}(K)\right| k_{1}^{-}\right\rangle=-\frac{1}{2 \pi} \frac{\delta\left(k_{1}^{\prime-}-k_{1}^{-}\right)}{\widehat{k}_{1}^{+}\left(K^{+}-\widehat{k}_{1}^{+}\right)\left(k_{1}^{-}-\frac{\widehat{\vec{k}}_{1 \perp}^{2}+m_{1}^{2}-i o}{\widehat{k}_{1}^{+}}\right)\left(K^{-}-k_{1}^{-}-\frac{\widehat{\vec{k}}_{2 \perp}^{2}+m_{2}^{2}-i o}{K^{+}-\widehat{k}_{1}^{+}}\right)},
$$

where operators are denoted by a "hat" and $\widehat{k}_{i o n}^{-}=\left(\widehat{\vec{k}}_{i \perp}^{2}+m^{2}\right) / \widehat{k}_{i}^{+}(i=1,2)$. The kinematical momentum states, $\left\langle x_{i}^{-} \vec{x}_{i \perp} \mid k_{i}^{+} \vec{k}_{i \perp}\right\rangle=e^{-\imath\left(\frac{1}{2} k_{i}^{+} x_{i}^{-}-\vec{k}_{i \perp} \cdot \vec{x}_{i \perp}\right)}$, are eigenfunctions of the free energy operator $\widehat{k}_{i o n}^{-}$. The completeness relation and the normalization are $\int \frac{d k^{+} d^{2} k_{\perp}}{2(2 \pi)^{3}}\left|k^{+} \vec{k}_{\perp}\right\rangle\left\langle k^{+} \vec{k}_{\perp}\right|=1$ and $\left\langle k^{\prime+} \vec{k}_{\perp}^{\prime} \mid k^{+} \vec{k}_{\perp}\right\rangle=2(2 \pi)^{3} \delta\left(k^{\prime+}-k^{+}\right) \delta\left(\vec{k}_{\perp}^{\prime}-\vec{k}_{\perp}\right)$.

The integrations over $k_{1}^{-}$and $k_{1}^{\prime-}$ eliminate the relative time: 


$$
g_{0}(K)=\left|G_{0}(K)\right|:=\int d k_{1}^{\prime-} d k_{1}^{-}\left\langle k_{1}^{--}\left|G_{0}(K)\right| k_{1}^{-}\right\rangle=i \widehat{\Omega}^{-1} g_{0}^{(2)}(K) \widehat{\Omega}^{-1},
$$

and the Fourier transform in $K^{-}$gives the free propagator in the $x^{+}$-time. Here, $K^{+}>0$ is used without any loss of generality. The phase space operator is $\widehat{\Omega}:=\sqrt{\widehat{k}_{1}^{+}\left(K^{+}-\widehat{k}_{1}^{+}\right)}$. The vertical bar $\mid$on the left of the Green's function represents integration on $k_{1}^{-}$in the bra-state, the bar on the right in the ket-state. The free two-body light-front Green's function is a particular case of the light-front Green's function for $N$ particles:

$$
g_{0}^{(N)}(K)=\left[\prod_{j=1}^{N} \theta\left(\widehat{k}_{j}^{+}\right) \theta\left(K^{+}-\widehat{k}_{j}^{+}\right)\right]\left(K^{-}-\widehat{K}_{0}^{(N)-}+i o\right)^{-1},
$$

where $\widehat{K}_{0}^{(N)-}=\sum_{j=1}^{N} \widehat{k}_{j \text { on }}^{-}$is the free light-front Hamiltonian.

For fermions, the Dirac propagator is written as $(\not k-m+i o)^{-1}=\left(k_{o n}+m\right) /\left(k^{2}-m^{2}+i o\right)+\gamma^{+} /\left(2 k^{+}\right)$, to separate the last term which is instantaneous in the $x^{+}$-time. Therefore, the free two-fermion propagator can be written as:

$$
G_{0}^{F}(K)=\left(\not k_{1}+m_{1}\right)\left(\not k_{2}+m_{2}\right) G_{0}(K)=\Delta G_{0}^{F}(K)+\bar{G}_{0}^{F}(K),
$$

where $\Delta G_{0}^{F}(K)$ contains the instantaneous terms and the global light-front time propagator of the two fermion system is $\bar{G}_{0}^{F}(K)=\left(k_{1 o n}+m\right)\left(k_{2 o n}+m\right) G_{0}(K)$. Here, we only define the basis states of spinorial functions in the positive energy sector as $\left\langle x^{-} \vec{x}_{\perp} \mid k^{+} \vec{k}_{\perp} s\right\rangle=e^{-i\left(\frac{1}{2} k^{+} x^{-}-\vec{k}_{\perp} \cdot \vec{x}_{\perp}\right)} u(k, s)$. The states form a complete basis for positive energy spinor functions,

$$
\sum_{s} \int \frac{d k^{+} d^{2} k_{\perp}}{2(2 \pi)^{3}}\left|k^{+} \vec{k}_{\perp} s\right\rangle\left\langle k^{+} \vec{k}_{\perp} s\right| \gamma^{0}=\mathbf{1}, u(k, s)=\frac{\left(k_{o n}+m\right)}{\sqrt{2 k^{+} 2 m}} \gamma^{+} \gamma^{0}\left(\begin{array}{c}
\chi_{s} \\
0
\end{array}\right) ;
$$

where $\chi_{s}$ is the two-component Pauli spinor and $u(k, s)$ is the light-front spinor [8]. We point out that one could equally well study particle-antiparticle systems or others.

Our aim here is to discuss the integral equation for the interacting light-front two-body propagator, $g(K)$, which is derived from

$$
G(K)=\bar{G}_{0}(K)+\bar{G}_{0}(K) T(K) \bar{G}_{0}(K),
$$

with $g(K) \equiv|G(K)|$. For a two-boson system $\bar{G}_{0}^{B}(K) \equiv G_{0}(K)$. We use the quasi-potential reduction of Ref. [10] to perform the projection to $x^{+}=0$. For that purpose an auxiliary Green's function $\widetilde{G}_{0}(K)$ is introduced as

$$
\widetilde{G}_{0}(K):=\bar{G}_{0}(K)\left|g_{0}^{-1}(K)\right| \bar{G}_{0}(K),
$$

which allows one to separate the light-front two-particle propagation in the intermediate states $[2,3]$. In our discussion, the operator $g_{0}^{-1}(K)$ for a two-fermion system is defined in the subspace of positive energy spinors.

The transition matrix is the solution of

$$
\begin{aligned}
& T(K)=W(K)+W(K) \widetilde{G}_{0}(K) T(K), \\
& W(K)=V(K)+V(K)\left[G_{0}(K)-\widetilde{G}_{0}(K)\right] W(K),
\end{aligned}
$$

according to Ref. [10]. The auxiliary three-dimensional transition matrix

$$
t(K)=g_{0}(K)^{-1}\left|\bar{G}_{0}(K) T(K) \bar{G}_{0}(K)\right| g_{0}(K)^{-1},
$$

gives the scattering amplitude for two-particles, which is identical to the one obtained directly from $T$ (see discussion in $[2,3])$. The auxiliary transition matrix satisfies

$$
t(K)=w(K)+w(K) g_{0}(K) t(K), w(K)=g_{0}(K)^{-1}\left|\bar{G}_{0}(K) W(K) \bar{G}_{0}(K)\right| g_{0}(K)^{-1} ;
$$

which can be derived using Eqs. (9) and (11). 


\section{HIERARCHY EQUATIONS FOR LF GREEN'S FUNCTIONS}

The two-body light-front propagator satisfies $g^{(2)}(K)=g_{0}^{(2)}(K)+g_{0}^{(2)}(K) \nu(K) g^{(2)}(K)$ where $g^{(2)}(K) \equiv-i \widehat{\Omega} g(K) \widehat{\Omega}$, $\nu(K)=i \widehat{\Omega}^{-1} w(K) \widehat{\Omega}^{-1}$, which is derived from Eqs. (7) and (12). The poles of $g^{(2)}(K)$ are at the positions of the bound states, and from its residue one can obtain the lowest Fock component of the wave function. In addition, the light-front description fully retrieves the covariant one [2].

The first order contribution to $\nu(K)$ obtained from Eqs. (10) and (12) is

$$
\nu^{(2)}(K)=i\left[\widehat{\Omega} g_{0}(K)\right]^{-1}\left|G_{0}(K) V(K) G_{0}(K)\right|\left[g_{0}(K) \widehat{\Omega}\right]^{-1} .
$$

The second order term in the quasi-potential expansion given by

$$
\begin{aligned}
\nu^{(4)}(K) & =i\left[\widehat{\Omega} g_{0}(K)\right]^{-1}\left|G_{0}(K) V(K) G_{0}(K) V(K) G_{0}(K)\right|\left[g_{0}(K) \widehat{\Omega}\right]^{-1} \\
& -i\left[\widehat{\Omega} g_{0}(K)\right]^{-1}\left|G_{0}(K) V(K) \widetilde{G}_{0}(K) V(K) G_{0}(K)\right|\left[g_{0}(K) \widehat{\Omega}\right]^{-1}
\end{aligned}
$$

is two-body irreducible, due to the subtraction of the second term in the r.h.s. of Eq. (14).

We restrict our discussion to the ladder approximation of the BSE. Below, we explicit the content of the operator $\nu^{(n)}$ in the light-front Fock-space. For the chosen bosonic Lagrangian model, the interaction operator acting between Fock-states differing by one quantum $\sigma$, has matrix element given by

$$
\left\langle q k_{\sigma}|v| k\right\rangle=-2(2 \pi)^{3} \delta\left(q+k_{\sigma}-k\right) \frac{g_{S}}{\sqrt{q^{+} k_{\sigma}^{+} k^{+}}} \theta\left(k_{\sigma}^{+}\right)
$$

where the delta function expresses the kinematical momentum conservation. The effective interaction $\nu(K)$ up to second order in $v$ is:

$$
\nu(K) \approx \nu^{(2)}(K)+\nu^{(4)}(K)=v g_{0}^{(3)}(K) v+v g_{0}^{(3)}(K) v g_{0}^{(4)}(K) v g_{0}^{(3)}(K) v,
$$

where, from Eq. $(13), \nu^{(2)}$ is $v g_{0}^{(3)}(K) v$. The contribution of four-body intermediate states to the three-body propagation arises from Eq.(14). In fact, $\nu(K)$ is equal to $v g^{(3)}(K) v$, which is found when the expansion is performed to all orders. From Eq.(16), one sees that $g^{(3)}$ is coupled to the four-body Green's function, which should be coupled to the five-body one, and so on. By generalization, one can construct a hierarchy of coupled equations for the Green's functions:

$$
\begin{aligned}
& g^{(2)}(K)=g_{0}^{(2)}(K)+g_{0}^{(2)}(K) v g^{(3)}(K) v g^{(2)}(K), \ldots \\
& g^{(N)}(K)=g_{0}^{(N)}(K)+g_{0}^{(N)}(K) v g^{(N+1)}(K) v g^{(N)}(K), \ldots
\end{aligned}
$$

In the Yukawa model for fermions, the interaction operator acting between Fock-states differing by zero, one and two $\sigma$ 's, has matrix elements given by

$$
\begin{aligned}
& \left\langle\left(q, s^{\prime}\right) k_{\sigma}|v|(k, s)\right\rangle=-2 m(2 \pi)^{3} \delta\left(q+k_{\sigma}-k\right) \frac{g_{S}}{\sqrt{q^{+} k_{\sigma}^{+} k^{+}}} \theta\left(k_{\sigma}^{+}\right) \bar{u}\left(q, s^{\prime}\right) u(k, s), \\
& \left\langle\left(q, s^{\prime}\right) k_{\sigma}^{\prime}|v|(k, s) k_{\sigma}\right\rangle=-2(2 \pi)^{3} \delta\left(q+k_{\sigma}^{\prime}-k-k_{\sigma}\right) \delta_{s^{\prime} s} \frac{g_{S}^{2}}{\sqrt{k_{\sigma}^{\prime+} k_{\sigma}^{+}}} \frac{\theta\left(k_{\sigma}^{\prime+}\right) \theta\left(k_{\sigma}^{+}\right)}{k^{+}+k_{\sigma}^{+}}, \\
& \left\langle\left(q, s^{\prime}\right) k_{\sigma}^{\prime} k_{\sigma}|v|(k, s)\right\rangle=-2(2 \pi)^{3} \delta\left(q+k_{\sigma}^{\prime}+k_{\sigma}-k\right) \delta_{s^{\prime} s} \frac{g_{S}^{2}}{\sqrt{k_{\sigma}^{++} k_{\sigma}^{+}}} \frac{\theta\left(k_{\sigma}^{++}\right) \theta\left(k_{\sigma}^{+}\right)}{k^{+}-k_{\sigma}^{+}} .
\end{aligned}
$$

The instantaneous terms in the two-fermion propagator give origin to Eqs. (19) and (20). The expansion of $\nu(K)$ has terms that couple sectors of the Fock-space that differ at most by two sigma's [3]. Therefore, the coupled set of Green's function found for bosons has to be extended for the Yukawa model as:

$$
\begin{aligned}
& g^{(2)}(K)=g_{0}^{(2)}(K)+g_{0}^{(2)}(K) v\left[g^{(3)}(K)+g^{(4)}(K)+g^{(3)}(K) v g^{(4)}(K)\right. \\
& \left.+g^{(4)}(K) v g^{(3)}(K)\right] v g^{(2)}(K), \ldots \\
& g^{(N)}(K)=g_{0}^{(N)}(K)+g_{0}^{(N)}(K) v\left[g^{(N+1)}(K)+g^{(N+2)}(K)+g^{(N+1)}(K) v g^{(N+2)}(K)\right. \\
& \left.+g^{(N+2)}(K) v g^{(N+1)}(K)\right] v g^{(N)}(K), \ldots
\end{aligned}
$$


The quasi-potential expansion differs from the truncation in the light-front Fock space. Taking the two-boson system as an example and restricting the intermediate state propagation up to four-particles, we find that $g^{(2)}(K)=$ $g_{0}^{(2)}(K)+g_{0}^{(2)}(K) v g^{(3)}(K) v g^{(2)}(K)$ and $g^{(3)}(K)=g_{0}^{(3)}(K)+g_{0}^{(3)}(K) v g_{0}^{(4)}(K) v g^{(3)}(K)$; which goes beyond the quasipotential expansion in second order, where, instead of $g^{(3)}(K)$, one has $g_{0}^{(3)}(K)$ (c.f. Eq. $\left.(16)\right)$.

\section{SUMMARY}

The set of coupled equations, (17) and (21), contains all two-body irreducible diagrams with the exception of those including closed loops in $\Phi_{1}$ and $\Phi_{2}$ or in $\Psi$ and as well part of the cross-ladder diagrams. The light-front Fock states contains two bosons $\Phi_{1}$ and $\Phi_{2}$ or two fermions with any number of bosons $\sigma$. This excludes the complete representation of the crossed ladder diagrams. The hierarchy set of equations also resembles the iterated resolvent method [8]. The covariant ladder restricts the kernel of the hierarchy equations, i.e., crossed terms and self energies are not included in Eqs. (17) and (21). This set of equations can also be useful in the study of renormalization in the light-front, as has been discussed for fermions in the context of the quasi-potential expansion [3].

In summary, we have presented the general framework for constructing the light-front two-body Green's function using a quasi-potential reduction of the Bethe-Salpeter equation. We have found a coupled set of equations which gives the two-body propagator in several cases, including the covariant ladder approximation. Finally, we have discussed within our scheme the truncation of the light-front Fock-space. Renormalization and local gauge invariance in this framework is a challenge open for future work.

We thank the Brazilian agencies FAPESP and CNPq for partial support.

[1] P.A.M. Dirac, Rev. Mod. Phys. 21 (1949) 392.

[2] J.H.O. Sales, T.Frederico, B.V. Carlson and P.U. Sauer, Phys. Rev. C61 (2000) 044003.

[3] J.H.O. Sales, T.Frederico, B.V. Carlson and P.U. Sauer, Phys. Rev. C63 (2001) 064003.

[4] T.Frederico, J.H.O. Sales, B.V. Carlson and P.U. Sauer, nucl-th/0301102, to appear in Few-Body Systems Supplements.

[5] S. Weinberg, Phys. Rev. 150 (1966) 1313.

[6] C.-R. Ji and R.J. Furnstahl, Phys. Lett. B167 (1986) 11; C.-R. Ji, Phys. Lett. B167 (1986) 16; C.-R. Ji, Phys. Lett. B322 (1994) 389.

[7] R.J.Perry, A. Harindranath and K.G. Wilson, Phys. Rev. Lett. 65 (1990) 2959; S.Glasek, A. Harindranath, S. Pinsky, J.Shigemitsu and K.G. Wilson, Phys. Rev. D47 (1993) 1599.

[8] S.J. Brodsky, H.-C. Pauli and S.S. Pinsky, Phys. Rep. 301 (1998) 299; and references therein.

[9] J. Carbonell, B.Desplanques, V.A. Karmanov and J.F. Mathiot, Phys. Rep.300 (1998) 215; M. Mangin-Brinet and J.Carbonell, Phys. Lett. B474 (2000) 237; J. R. Cooke, G. A. Miller, D. R. Phillips, Phys. Rev. C61 (2000) 064005; M. Mangin-Brinet, J. Carbonell V.A. Karmanov, Phys. Rev. D64 (2001) 125005; B. C. Tiburzi and G. A. Miller, Phys. Rev. D65 (2003) 074009.

[10] R.M. Woloshyn and A.D.Jackson, Nucl. Phys. B64 (1973) 269. 